\title{
Patient-related Barriers to Pain Management among Cancer Patients
}

\author{
Mai Goblan ${ }^{1}$, Mohammad ALBashtawy ${ }^{2}$, Omar Al Omari $^{3}$, Omar Khraisat ${ }^{4}$, Ahmad AL-Bashaireh ${ }^{5}$, \\ Zaid AlBashtawy ${ }^{6}$, Abdullah Alkhawaldeh ${ }^{7}$ \\ ${ }^{1}$ Master Student, Critical Care Nursing, Princess Salma Faculty of Nursing, AL al-Bayt University, Mafraq, \\ Jordan, ${ }^{2}$ Professor, Princess Salma Faculty of Nursing, AL al-Bayt University, Mafraq, Jordan, \\ ${ }^{3}$ Associate Professor, Faculty of Nursing, Sultan Qaboos University, Muscat, Oman, ${ }^{4}$ Assistant Professor, Faculty \\ of Nursing, Al-Ahliyya Amman University, Amman, Jordan, ${ }^{5}$ Assistant Professor, Faculty of Nursing, Al-Ahliyya \\ Amman University, Amman, Jordan, ${ }^{6}$ Faculty of Medicine, Yarmouk University, Irbid, Jordan, ${ }^{7}$ Assistance \\ Professor, Princess Salma Faculty of Nursing, AL al-Bayt University, Mafraq, Jordan
}

\begin{abstract}
Recently, despite the development of cancer pain treatment, many cancer patients still suffer from pain. This review aimed to explore the patients-related barriers to pain management among cancer patients. The electronic searching was conducted in a different database: Google Scholar, CINAHL and PubMed. According to previous studies barriers can classified into patients/family- related barriers, health care provided-related barriers, and institutional-related barriers. Patient-related barriers were reluctant to report pain, fear from tolerance, addiction, and side effect, and culture. This review provides summary data about the patient-barriers to effective cancer pain control.
\end{abstract}

Keywords: Cancer Pain, Barriers, Pain Management.

\section{Introduction}

In 2016, about 15.5 million persons with cancer were alive in the United Strat (US) and, by 2026 that total is likely to increase to almost 20 million ${ }^{(1)}$. Pain is considered one of the common symptoms in cancer patients and can be initiated by cancer itself, surgery, treatment, treatment side, tests and procedures (2). Studies proposed that pain occurs in about $50 \%$ of cancer survivor's patients ${ }^{(3,4)}$.

There are effective methods to avoid and control pain in and after cancer treatment; quick identification of pain signs, communication and classification regarding pain type and severity, pharmacologic and non-pharmacologic pain control choices and education

\section{Corresponding Author:}

Abdullah Alkhawaldeh, $\mathrm{PhD}$, Assistance Professor, Princess Salma Faculty of Nursing, AL al-Bayt University, Mafraq, Jordan.

Email: dr-abd@aabu.edu.jo, abdo_752012@yahoo.com of patient $^{(5)}$

Recently, despite the development of cancer pain treatment in terms of surgical, drug and non-drug interventions, many cancer patients still suffer from pain ${ }^{(6,7)}$, about $30 \%$ of cancer patients do not obtain pain medication comparative to their amount of pain (8). The untreated pain still a major feared consequences of cancer ${ }^{(9,10,11)}$. It affected on physical, functioning, psychological well-being and social interaction of patients ${ }^{(12,13,14)}$. In Jordan the prevalence of pain among Jordanian cancer patients is high ${ }^{(15)}$.

This study aimed to explore the patients related barriers to pain management among cancer patients.

\section{Method}

Search methods: The electronic searching was conducted in a different database: Google Scholar, CINAHL and PubMed. Key search terms used: cancer pain and barriers. 
The studies that focused on the topic of Aortic valve stenosis, were published in English between 2000 and 2018 were included in the review. While, studies published before 2000 were excluded.

Search outcome: Exploring of literature yield about 50 studies for review, after reading the studies about 35 study were excluded and 15 studies met the inclusion criteria.

\section{Discussion}

In the literature, the barriers of pain management among cancer patients were classified into three groups: (1) patients related barriers, (2) physician related barriers, and (3) institutional and health care system related barriers (16). Patient related barriers were patient reluctance to report pain and adhere to treatment recommendations. Besides, cognitive, affective and sensory patientrelated barriers to cancer pain management with opioid analgesics. Physician related barriers were insufficient physicians' knowledge about cancer pain management, inadequate patterns of pain assessment, and inadequate opioid prescription. Institutional and health care system related barriers were relevant only in countries with restrictive opioid prescription regulations ${ }^{(16)}$.

A cross-sectional study was conducted to identify the barriers to effective cancer pain control in patients and their families in Jordan. The study indicated a four main barriers to pain control: communication concerns, fears connected to addiction, side effects and fatalistic beliefs ${ }^{(17)}$. Another cross-sectional study was conducted to investigated barriers of pain management in Australian patients. The data indicated that patients fear of opioid drug addiction, tolerance, and side effect. Patient reduce dose of opioids drug because they fear side effect such as constipation. Also, they fear from needle injection so they seek alternative therapies such as massage, acupuncture, herbal remedies and heat ${ }^{(18) .}$

In Turkey, a cross-sectional study was conducted to define the patient-related barriers to cancer pain management. The study found that patients have high scores of misconception regarding pain and pain management. Also, patients did not report their pain because they fear from medicine ${ }^{(19)}$. In USA, a study was carried out to search barriers to pain management in African American and Hispanic cancer patients. The data reflected that the majority of patients expressed a concerns regarding the possible addiction to opioid drugs and the tolerance development. Also, the patients defined their physicians as the most common and trusted source of data about cancer pain ${ }^{(20)}$.

Patients' perceived barriers to handling cancer pain can be influenced by culture ${ }^{(21-30)}$. A recent qualitative study aimed to discover the patient's barriers to cancer pain management from the viewpoint of cancer patients and their family. The data reflected that the main barriers to effective cancer pain management were: knowledge deficit, regulatory factors and the use of cultural and religious approaches to manage pain (21). Another study was conducted to compared differences in Asian and Western patient-perceived barriers to handling cancer pain. That study indicated that Asian patients' perceived barriers to handling cancer pain were significantly greater than those for Western patients (particularly for concerns regarding disease progression, fatalism and tolerance) ${ }^{(24)}$.

In Korea, a study confirmed poor knowledge and inappropriate practices among physicians and nurses regarding pain management ${ }^{(31)}$. Studies have revealed that nurses have knowledge gaps regarding pain management, which negatively impact the quality of pain control in cancer patients ${ }^{(32) .}$ Nurses' knowledge about opioids' role in pain management was also reported as low by a study conducted in Italy, highlighting their irrational fear of the opioids' potential to result in addiction or respiration inhibition ${ }^{(33)}$.

\section{Conclusion}

The barriers of pain management among cancer patients were classified into three groups. Patient-related barriers were reluctant to report pain, fear from tolerance, addiction, and side effect, and culture. Study confirmed poor knowledge and inappropriate practices among physicians and nurses regarding pain management. This review provides summary data about the barriers to effective cancer pain control.

Conflict of Interest: No conflict of interest.

Ethical Clearance: Taken from Princess Salma Faculty of Nursing, AL al-Bayt University ethical committee. 
Source of Funding: Self.

\section{References}

1. Bluethmann SM, Mariotto AB, Rowland JH. Anticipating the "Silver Tsunami": prevalence trajectories and comorbidity burden among older cancer survivors in the United States. Cancer Epidemiol Biomarkers Prev 2016; 25(7):1029-36.

2. Levy MH, Chwistek M, Mehta RS. Management of chronic pain in cancer survivors. Cancer J 2008; 14(6):401-9.

3. Van den Beuken-van Everdingen, M. H. J., et al. "Prevalence of pain in patients with cancer: a systematic review of the past 40 years." Annals of oncology 2007; 18:1437-1449.

4. Khatatbeh, M., Jadallah, K., ALBashtawy, M., Malkawi, S., Gharaibah, M., Baslika, N. \& Smadi,W . (2017). Factors associated with colorectal cancer among Jordanians; A case-control study. Accepted at 27/12/2018 in Asian Pacific Journal of Cancer Prevention.

5. Chang KL, Fillingim R, Hurley RW, Schmidt S. Chronic pain management: nonpharmacological therapies for chronic pain. FP Essent 2015; 432:216.

6. Karine Azevedo São Leão Ferreira, Miako Kimura, Manoel Jacobsen Teixeira. The WHO analgesic ladder for cancer pain control, twenty years of use (2006). How much pain relief does one get from using it? Support Care Cancer 2006; 14:1086-1093.

7. Abu obead, k, Batiha A, Al-Jauissy, M, Alhalaiqa, F. \& ALBashtawy, M. Impact of radiotherapy treatment on Jordanian cancer patients' quality of life and fatigue. International Journal of Advanced Nursing Studies 2013; 3:6-12.

8. Greco MT, Roberto A, Corli O, Deandrea S, Bandieri E, Cavuto S, et al. Quality of cancer pain management: an update of a systematic review of undertreatment of patients with cancer. J Clin Oncol 2014; 32:4149-54.

9. Judith A. Paice (2018). Cancer Pain Management and the Opioid Crisis in America: How to Preserve Hard-Earned Gains in Improving the Quality of Cancer Pain Management. Cancer.

10. Freij, M, Al Qadire, M, Khadra, M, ALBashtawy,
M, Tuqan, W, Al Faqih,M , Innabi, A, Batiha, A-M, Alhalaiqa, F, and El-Razek, A. Awareness and Knowledge of Ovarian Cancer Symptoms and Risk Factors: A Survey of Jordanian Women. Clinical Nursing Research 2017, 1-15.

11. Al Qadire,M ., et al. "Predictors of anxiety and depression among parents of children with cancer in Jordan." Psychooncology 2018; 27:1344-6. †

12. Batiha, A, Abu Obead, K, Alhalaiqa, F, Kawafha, M, Abd EL-Razek,A, ALBashtawy, M., Saifan,A, Abu Ruz, M, Al ewaidat, H. Quality of Life and Fatigue among Jordanian Cancer Patients. Iranian Journal of public health 2015; 44:1704-1705.

13. Victoria T. Potter, C. Elke Wiseman, Stewart M. Dunn, y and Frances M. (2003). Patient Barriers to Optimal Cancer Pain Control. Psycho-Oncology $2003 ; 12: 153-160$.

14. Howeri, N., AlBashtawy, M., Ozaybi, N., Alkhawaldeh, A., Al Azzam, M., Albashtawy, S., Abdalrahim, A., Khraisat, O., AlBashtawy Z., and Suliman M. The Role of Ketogenic Diet in the Treatment of Cancer: A Review Paper. EC Psychology and Psychiatry 2020; 9:01-03.

15. Al Qadire, M., Tubaishat, A., Aljezawi MM. Cancer pain in Jordan: prevalence and adequacy of treatment. International Journal of Palliative Nursing 2013; 19.

16. Ramunë Jacobsen, Zita Liubarskienë1, Claus M,ldrup, Lona Christrup, Per $\mathrm{Sj}$,gren , Jurgita Samsanavièienë. Barriers to cancer pain management: a review of empirical research Medicina (Kaunas) 2009; 45.

17. Saifan, Ahmad, et al. Patient-and family caregiverrelated barriers to effective cancer pain control. Pain management nursing 2015; 16:400-410.'

18. Potter, Victoria T., et al. "Patient barriers to optimal cancer pain control." Psycho-Oncology: Journal of the Psychological, Social and Behavioral Dimensions of Cancer 2003; 12:153-160.

19. Bağçivan, Gülcan, et al. Analysis of patient-related barriers in cancer pain management in Turkish patients. Journal of pain and symptom management 2009, 38:727-737.'

20. Anderson KO, Richman SP, Hurley J, Palos G, Valero V, Mendoza TR, Gning I, Cleeland CS. 
Cancer pain management among underserved minority outpatients: perceived needs and barriers to optimal control. Cancer 2002; 94:2295-304.

21. Al-Ghabeesh, Suhair Hussni, et al. Barriers to effective pain management in cancer patients from the perspective of patients and family caregivers: A qualitative study. Pain Management Nursing 2020; 21:238-244.

22. Al Qadire, M., Alkhalaileh, M., ALBashtawy, M. (2018). Lifestyle and Dietary Factors and Prostate Cancer Risk: A Multicentre Case-Control Study. Clinical Nursing Research 2018.

23. Alkhawaldeh, A, ALOmari,O, ALBashtawy,M, Aljezawi, M, Suliman, M, Holm, M, Qaddumi, J, Batiha, A-M, Alhalaiqa, F, \& Saifan , A. Longterm conditions associated with primary health care service use among older adults. Primary Health Care 2016; 26:31-35.

24. Chen, Chen Hsiu, Siew Tzuh Tang, and Chien Hao Chen. "Meta-analysis of cultural differences in Western and Asian patient-perceived barriers to managing cancer pain." Palliative Medicine 26.3 (2012): 206-221.

25. ALBashtawy, M., Gharaibeh, H., Alhalaiqa, F., Batiha,AM.,, Freij.,M., Saifan , A, Al- alwamreh, K., Hamadneh. SH., Al-Kloub, M. \& Khamaiseh, A. The Health Belief Model's Impacts on the Use of Complementary and Alternative Medicine by Parents or Guardians of Children with Cancer. Iran J Public Health 2016; 45: 708-709.

26. Suliman, M,. Aloush, S,. Aljezawi, M. \& ALBashtawy, M. Knowledge and practices of isolation precautions among nurses in Jordan. American Journal of Infection Control 2017.
27. ALBashtawy M., et al. "Assessment of headache among high school students in Jordan.” The Journal of School Nursing 2019; 35:88-95.ๆ

28. Al Qadire, Mohammad, et al. "Public Attitudes toward Cancer and Cancer Patients: A Jordanian National Online Survey." Middle East Journal of Cancer (2020).

29. Sulaiman, M.\& Aljezawi, M., ALBashtawy, M., Fitzpatrick, J., Aloush . S. \& Al-Awamreh, k Exploring Safety Culture in Jordanian Hospitals: A Baseline Study". Journal of Nursing Care Quality 2017; 32:p E1-E7.

30. Aloush, S.M., Al-Sayaghi, K., Tubaishat, A., Dolansky, M., Abdelkader, F.A., Suliman, M., ALBashtawy, M., Halabi, M. Compliance of Middle Eastern hospitals with the central line associated bloodstream infection prevention guidelines. Applied Nursing Research 2018; 43:5660.

31. Omran S, Al Qadire M, Ali NA, Al Hayek MF. Knowledge and attitudes about pain management: a comparison of oncology and non-oncology Jordanian nurses. Nurs Health. 2014; 2:73-80.

32. Jho HJ, Kim Y, Kong KA, Kim DH, Choi JY, Nam EJ, Choi JY, Koh S, Hwang KO, Baek SK, et al. Knowledge, practices, and perceived barriers regarding cancer pain management among physicians and nurses in Korea: a nationwide multicenter survey. PLoS One 2014; 9:e105900.

33. Bernardi M, Catania G, Lambert A, Tridello G, Luzzani M. Knowledge and attitudes about cancer pain management: a national survey of Italian oncology nurses. Eur J Oncol Nurs. 2007;11(3):272-9. 